\title{
Análisis de datos de Radares Meteorológicos en el territorio Colombiano para el diagnóstico y detección de errores
}

\section{Meteorological Radar data analysis in Colombian Territory for diagnostics and error detection}

\author{
Ernesto Gomez Vargas¹.; Gustavo Robayo².;Julián Camargo³.; Laura Cadena ${ }^{4}$
}

\footnotetext{
${ }^{1}$ Coordinador de Maestría y Docente, Universidad Distrital FJC. egomezvargas@gmail.com

2 Magister, Universidad Distrital FJC. tavoalejo@gmail.com

${ }^{3}$ Docente, Universidad Distrital FJC. jcamargo15@gmail.com

${ }^{4}$ Magister, Universidad Distrital FJC. lacadenacontreras@gmail.com
}

\section{Resumen}

Se realiza el análisis de entrada sobre los datos de dos radares meteorológicos en Colombia. Se muestra la decodificación y transformación de los datos necesaria para una caracterización estadística de los principales observables polarimétricos. Este resultado permite hacer unas consideraciones $y$ conclusiones importantes para detección y posible corrección de errores en las mediciones de radar.

Palabras clave: clasificador de hidrometeoros, observables polarimétricos, radar meteorológico.

\begin{abstract}
Entry analysis of colombian meteorological radars data is performed. Decoding and transforming is shown and statistical characterization of main polarimetric observables. This result is basis for concuding some important aspects about detection and possible correction of error in radar measurement.
\end{abstract}

Keywords: meteorological radar, hydrometeor classifier, polarimetric observables. 
57

\section{Introducción}

El radar meteorológico polarimétrico es un instrumento de medición utilizado para la detección de la precipitación y otros fenómenos meteorológicos. Está basado en radiaciones de ondas electromagnéticas que luego son reflejadas en los cuerpos en el espacio (blancos). La energía reflejada por los blancos es recibida por el radar y convertida en datos digitales para ser procesados por un software especializado (Socorras Quintero, Gomez Vargas, \& Obregon Neira, 2014). Los métodos aplicados en Colombia para clasificación de hidrometeoros y posterior estimación de precipitación, están arrojando resultados con altos márgenes de error. En una exploración inicial, se observa que las clasificaciones erróneas obedecen a varios factores, como la obsolescencia de algoritmos utilizados y aspectos debidos al radar como la atenuación de propagaciones anómalas, ecos terrestres, la condensación, la evaporación y la calibración del radar (Gomez Vargas \& Obregon Neira, 2015). Se requiere el desarrollo de algoritmos y módulos informáticos para realizar un procesamiento de los datos de radares que se tienen disponibles. Este procedimiento debe utilizar los denominados observables polarimétricos: Reflectividad diferencial (Zdr), diferencial de fase (Kdp) y otros que entrega el radar, involucrar las características de la geografía cercana y considerar las fuentes de error mencionadas.

En este documento se mostrará el análisis realizado sobre los observables polarimétricos de dos de los radares ubicados en Colombia (Tablazo y Corozal). El análisis permite caracterizar las variables polarimétricas entregadas por el radar y determinar los posibles errores que están afectando las mediciones y así dar bases para proponer métodos de corrección de errores.

En las secciones posteriores se describirán los parámetros de los dos radares y los eventos que se analizaron, el tratamiento necesario de los datos y el tratamiento realizado para obtener la caracterización estadística de los datos.

Finalmente se realizan consideraciones sobre la caracterización estadística de los datos y sobre el clasificador de hidrometeoros del fabricante de los radares.

\section{Procedencia de los datos y decodificación}

Las mediciones a analizar fueron registradas por 2 radares ubicados en el territorio colombiano, de cada uno de ellos se extrajeron los datos de 2 eventos de precipitaciones. A continuación las características de cada radar y de los eventos seleccionados.

Los datos deben ser tratados para poderlos visualizar y procesar para su análisis. De toda la información que entrega el radar, interesan los observables polarimètricos de la tabla 4 y 5 en donde se muestra la 
parametrización de cada uno de los observables.

Tabla 1. Parámetros de Radares.

\begin{tabular}{|c|c|c|}
\hline Ubicación & Tablazo & Corozal \\
\hline Banda & $\mathrm{C}$ & $\mathrm{C}$ \\
\hline Num PPI & 10 & 10 \\
\hline Longitud & $-74,2037$ & $-75,28299$ \\
\hline Latitud & 5,01180008 & 9,3309999 \\
\hline Altitud & 3544 metros & 143 metros \\
\hline Frec. & $5,6246241 \mathrm{MHz}$ & $5,6246241 \mathrm{MHz}$ \\
\hline $\begin{array}{c}\text { Tamaño de } \\
\text { Celda }\end{array}$ & 450 metros & 450 metros \\
\hline $\begin{array}{l}\text { Total } \\
\text { Celdas }\end{array}$ & 239040 & 239040 \\
\hline
\end{tabular}

Tabla 2. Eventos Radar Tablazo

\begin{tabular}{|c|c|c|c|}
\hline \multicolumn{4}{|c|}{ Radar Tablazo } \\
\hline Cod. & Fecha & Desc. & $\begin{array}{c}\text { \% de celdas con } \\
\text { Precipitación }\end{array}$ \\
\hline TAB2 & $13 / 10 / 12$ & Lluvia & 41,92 \\
\hline$T A B 3$ & $18 / 10 / 12$ & Lluvia & 52,55 \\
\hline
\end{tabular}

Tabla 3. Eventos Radar Corozal

\begin{tabular}{|c|c|c|c|}
\hline \multicolumn{4}{|c|}{ Radar Corozal } \\
\hline Cod. & Fecha & Desc. & $\begin{array}{c}\text { \% de celdas con } \\
\text { Precipitación }\end{array}$ \\
\hline COR2 & $28 / 06 / 13$ & $\begin{array}{c}\text { Lluvia y } \\
\text { granizo }\end{array}$ & 48,7 \\
\hline COR3 & $11 / 07 / 13$ & Lluvia & 54,89 \\
\hline
\end{tabular}

Tabla 4. Observables polarimétricos Corozal

\begin{tabular}{|c|c|c|c|c|c|}
\hline \multicolumn{6}{|c|}{ Radar Corozal } \\
\hline ID & Var. & units & FillValue & $\begin{array}{l}\text { scale_ } \\
\text { factor }\end{array}$ & $\begin{array}{c}\text { add_ } \\
\text { offset }\end{array}$ \\
\hline 94 & DBZ & $\mathrm{dBZ}$ & -128 & 0.5000 & 32 \\
\hline 96 & ZDR & $\mathrm{dB}$ & -128 & 0.0625 & 0 \\
\hline 97 & KDP & $\mathrm{deg} / \mathrm{km}$ & -128 & 1 & 128 \\
\hline
\end{tabular}

Tabla 5. Observables Polarimétricos Tablazo

\begin{tabular}{|c|c|c|c|c|c|}
\hline \multicolumn{7}{|c|}{ Radar Tablazo } \\
\hline ID & Var. & units & FillValue & $\begin{array}{l}\text { scale_ } \\
\text { factor }\end{array}$ & $\begin{array}{c}\text { add_ } \\
\text { offset }\end{array}$ \\
\hline 94 & DBZ & $\mathrm{dBZ}$ & -128 & 0.5000 & 32 \\
\hline 96 & ZDR & $\mathrm{dB}$ & -128 & 0.0625 & 0 \\
\hline 97 & $\begin{array}{c}\text { DBZ_- } \\
\text { CORR }\end{array}$ & $\mathrm{dBZ}$ & -128 & 0.5000 & 32 \\
\hline 98 & $\mathrm{KDP}$ & $\mathrm{deg} / \mathrm{km}$ & -128 & 1 & 128 \\
\hline
\end{tabular}

Finalmente se realiza una transformación de cada variable polarimétrica de acuerdo al flujograma de la figura 1 , esto es necesario para obtener el valor real de cada una.

\subsection{Sobre el clasificador de Hidrometeoros}

Adicional a las variables mostradas, se transformaron las variables que corresponden al clasificador. Se obtienen valores enteros entre 0 y 255 que se deben decodificar para su respectiva interpretación. Se realiza la conversión de base 10 a binario obteniendo así un código binario de 8 bits. Los dos primeros bits corresponden a una clasificación (C2), los siguientes tres a otra (C1) y los últimos tres a otra (C3). La estructura del clasificador del IRIS se muestra en la Figura 2. 


\begin{tabular}{|c|c|c|c|c|}
\hline \multicolumn{2}{|r|}{$C 1$} & cultad de Ciencias Básicas.2018.16(1) & $5-66$ & \multirow{2}{*}{$\underset{\mathrm{fcb}}{\Rightarrow}$} \\
\hline Valor & Clase & 011(3) & Nieve Húmeda & \\
\hline $000(0)$ & No disponible & & & \\
\hline $001(1)$ & $\begin{array}{l}\text { Eco de tierra propagación } \\
\text { anómala }\end{array}$ & $101(5)$ & Graupel & \\
\hline $010(2)$ & Eco biológico & $110(6)$ & Granizo & \\
\hline 011(3) & Precipitación & $111(7)$ & No usado & \\
\hline
\end{tabular}

Tabla 7. Interpretación clasificadoR C1

\begin{tabular}{|l|l|}
\hline $101(5)$ & Precipitación liviana \\
\hline $110(6)$ & Precipitación moderada \\
\hline $111(7)$ & No usado \\
\hline
\end{tabular}

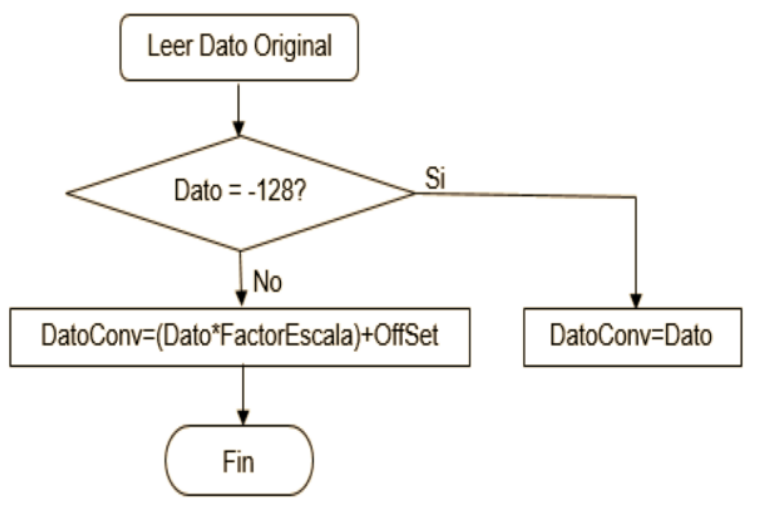

Figura 1. Flujograma transformación de datos

\begin{tabular}{|c|ccc|c|c|c|c|}
7 & 6 & 5 & 4 & 3 & 2 & 1 & 0 \\
\hline $\mathrm{C} 2$ & & & $\mathrm{C} 1$ & & & \multicolumn{2}{c}{$\mathrm{C} 0$} \\
\hline
\end{tabular}

Figura 2. Decodificación del valor binario del clasificador

Las tablas 6, 7 y 8 muestran la interpretación de los diferentes valores que pueden tomar los clasificadores, $\mathrm{C} 0, \mathrm{C} 1$ y C2.

Tabla 6. Interpretación clasificador C0

\begin{tabular}{|c|lc|}
\hline \multicolumn{2}{|c|}{ CO } \\
\hline Valor & \multicolumn{1}{|c|}{ Clase } \\
\hline $000(0)$ & No disponible \\
\hline $001(1)$ & $\begin{array}{l}\text { Blanco } \\
\text { meteorológico }\end{array}$ \\
\hline $010(2)$ & Lluvia \\
\hline
\end{tabular}

3. Análisis de los datos

\subsection{Visualización de reflectividad} Utilizando los valores obtenidos de $\mathrm{dbZ}$ para cada ángulo y celda se graficó la reflectividad para cada evento. Para la gráfica se utilizó la escala de colores (figura 3). 
60

Se muestran en las figuras 4, 5, 6, 7 los eventos que muestran la mayor cantidad de celdas con precipitación moderada. Para elaborar las gráficas 4 y 5 se utilizaron los datos del radar Corozal (eventos 2 y 3 ).

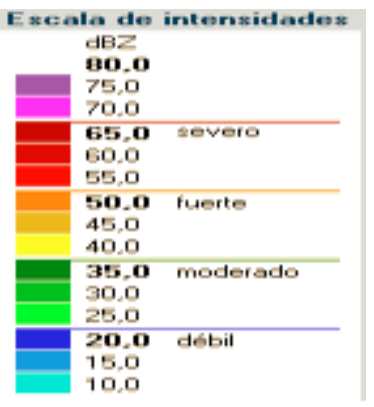

Figura 3. Código de colores reflectividad de radar. Fuente: (Rinehart, 2007)

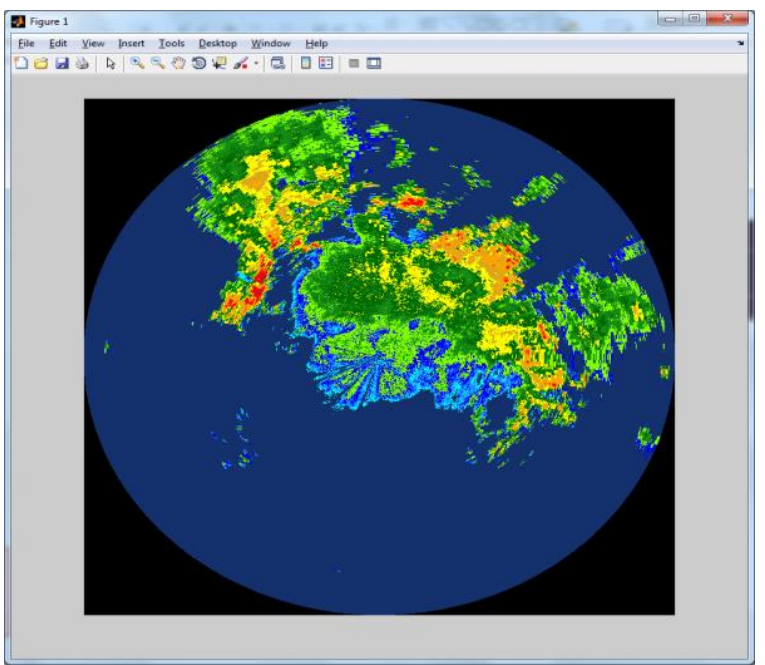

Figura 4. Evento COR2

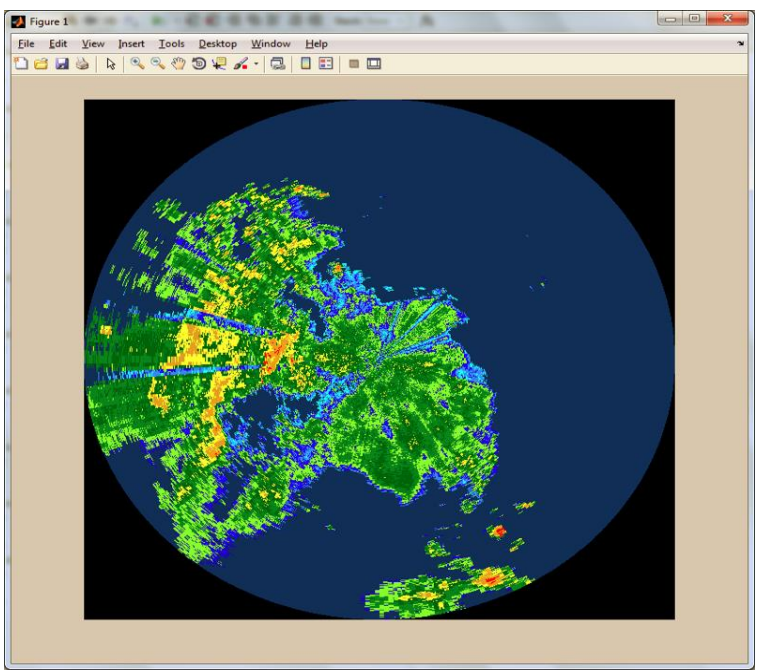

Figura 5. Evento COR3

Para elaborar las gráficas 6 y 7 se utilizaron los datos de radar Tablazo (eventos 2 y 3 ). 


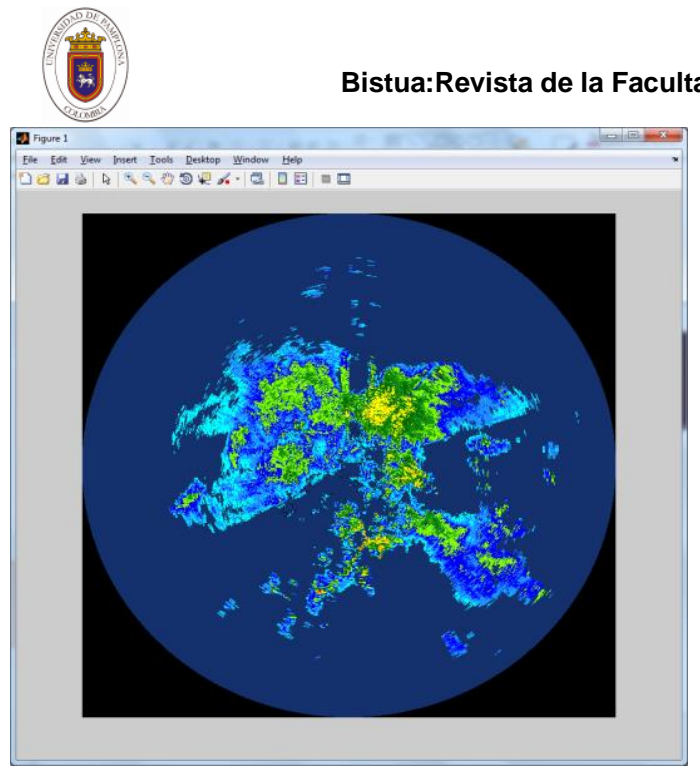

Figura 6. Evento TAB2

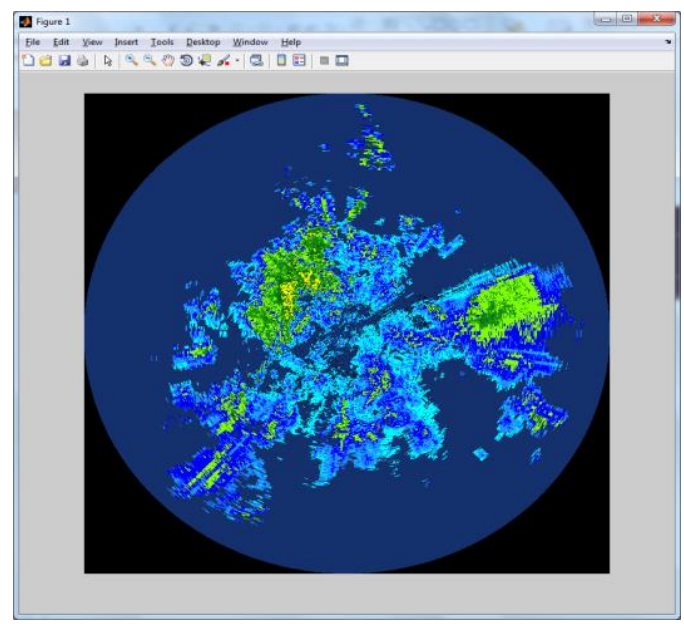

Figura 7. Evento TAB3

\subsection{Análisis Estadístico}

Para cada uno de los observables polarimétricos se muestran los histogramas de los eventos seleccionados, a partir de esto se realizarán unas consideraciones importantes para el objetivo del presente trabajo.

Tabla 9. Histogramas de la Reflectividad

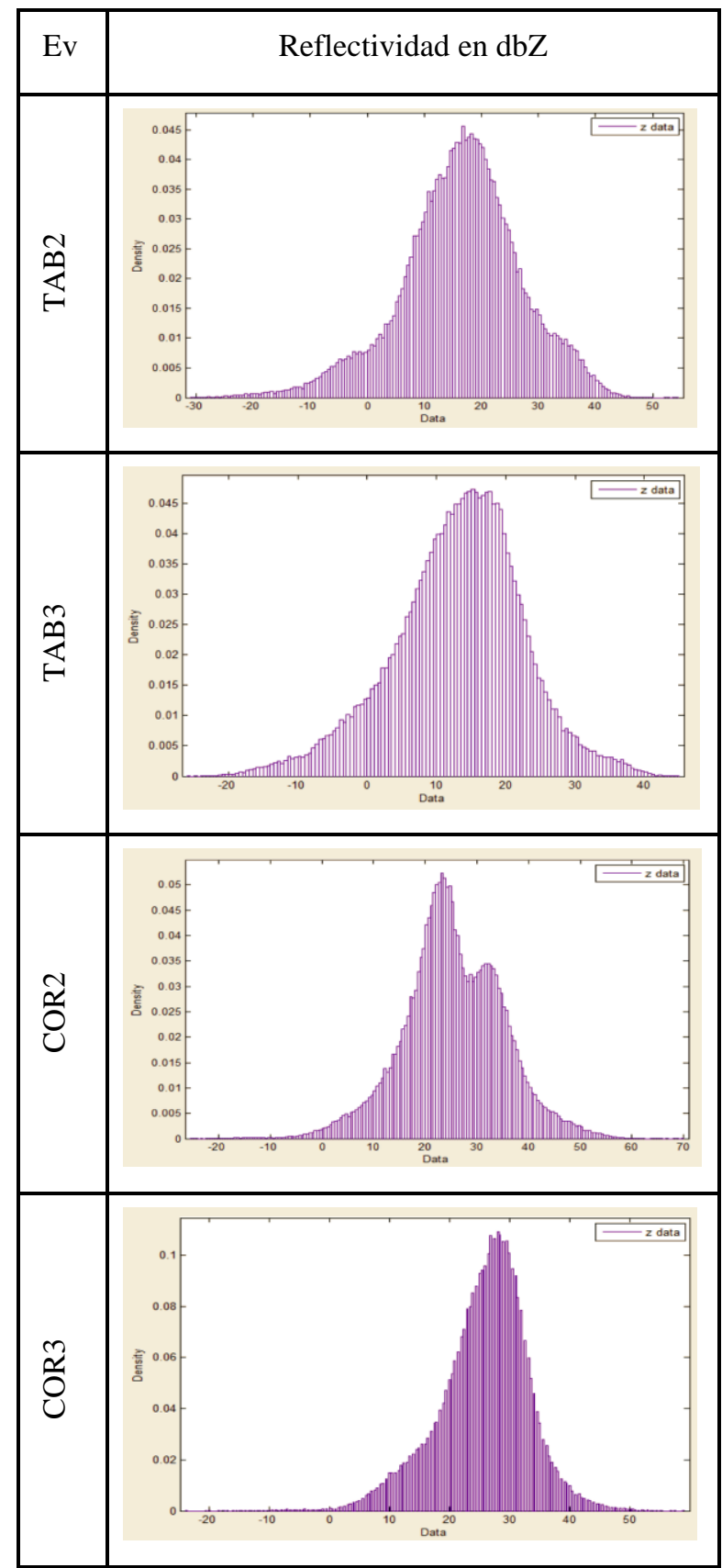

61 
Bistua:Revista de la Facultad de Ciencias Básicas.2018.16(1):56-66

Tabla 10. Histogramas de ZDR

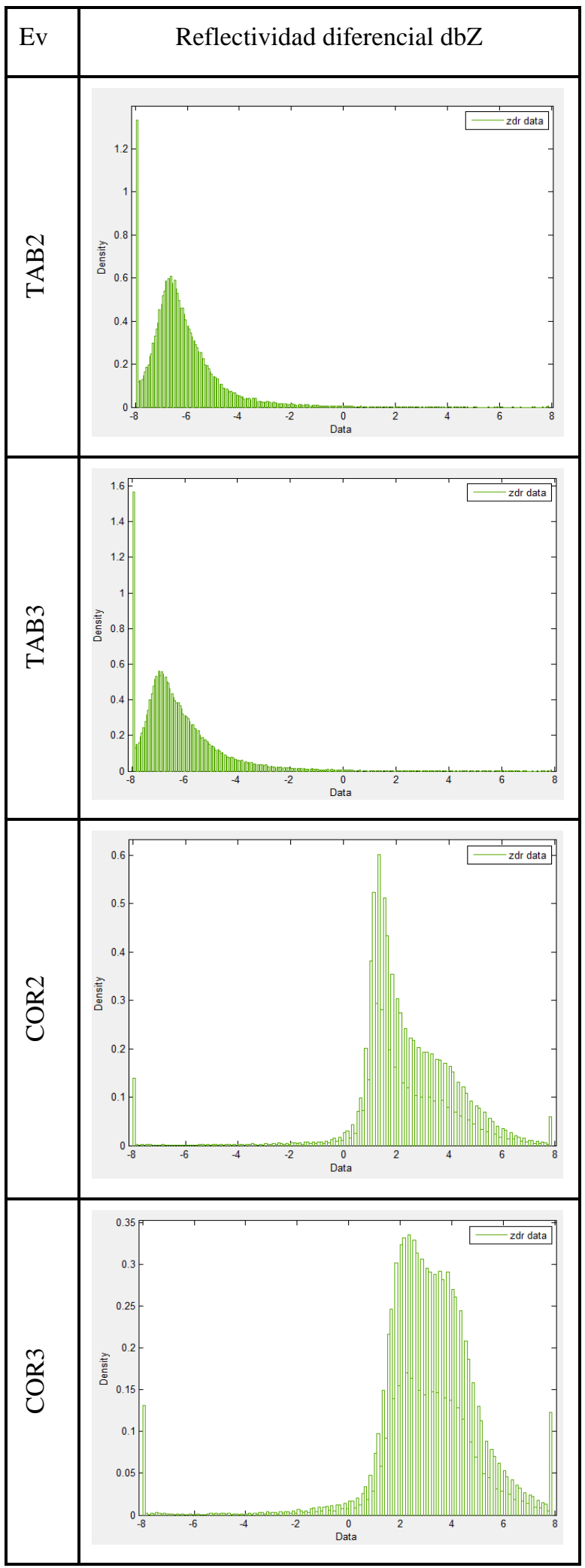

Tabla 11. Histogramas de KDP

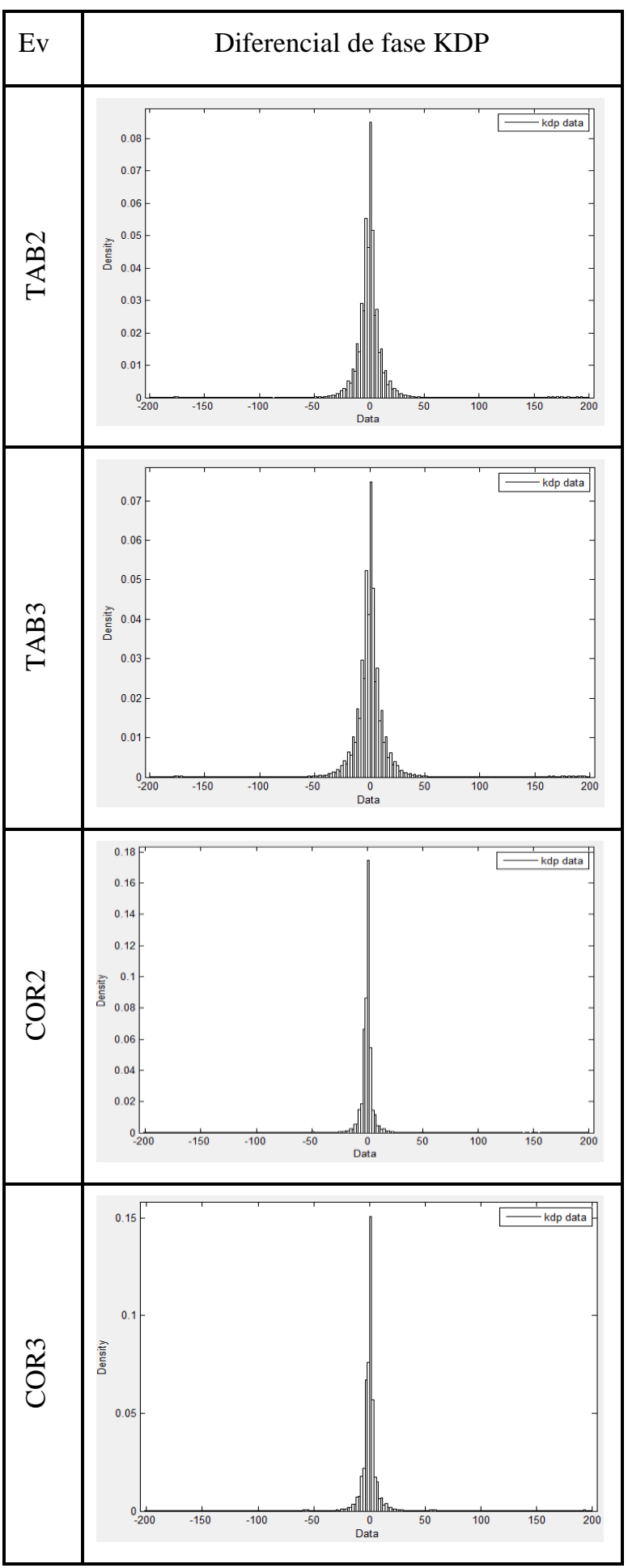

62 
De la misma forma es posible generar los histogramas para las demás variables polarimétricas de interés. En la Tablas 11 y 12 se resumen algunos valores de interés para las diferentes variables polarimétricas de los eventos de precipitación del Radar de Tablazo, a partir de esas es posible hacer algunas consideraciones importantes sobre la confiabilidad de los datos relacionados con la investigación puntual.

\subsection{Consideraciones Radar Tablazo}

Se observa que los valores de $Z_{D R}$ son negativos. Esto implicaría que la mayoría de hidrometeoros son más altos que anchos (Bringi, Thurai, \& Hannesen, 2007), característica que bien podría darse en hidrometeoros como el Graupel (cristales con de hielo con forma de cono) que no son posibles en una geografía como la Colombiana, razón por la cual se puede concluir que se presenta algún tipo de problema en la configuración del radar Tablazo que hace que esta variable se esté midiendo o almacenando de manera incorrecta.

También se evidencia que la variable KDP tiene una desviación estándar más alta que la de radar corozal. Es decir, que si bien la media está cerca a cero, hay una cantidad apreciable de valores por fuera de los estándares. En consecuencia se puede concluir que existe algún problema de configuración o de almacenamiento en el radar el tablazo con estas dos variables.
63

La distribución de la variable $Z$ de los diferentes eventos del Radar de Tablazo, muestra información está concentrada en una zona que se considera con presencia de lluvia de diversos tipos (Lluvia liviana, moderada y pesada), pero con muy poca presencia de granizo.

\subsection{Consideraciones Radar Corozal}

Se observa en la tabla 13 que el ZDR en este radar para ambos eventos es positivo. Para las variables ZDR y HV en ambos eventos del radar de Corozal su distribución es leptocúrtica teniendo un alto grado de concentración de los valores centrales de cada variable. Para Z y ZDR se tiene una distribución asimétrica negativa (Moda>Mediana>Media). KDP tienen una distribución asimétrica positiva (Media>Mediana>Moda).

Tabla 12. Caracterización estadística de los observables radar Tablazo

\begin{tabular}{|l|l|l|l|}
\hline $\begin{array}{l}\text { Evento } \\
\text { TAB2 }\end{array}$ & $Z h$ & $Z D R$ & Kdp \\
\hline Media & 16,0833 & $-6,1781$ & 0,192 \\
\hline Mediana & 16,5 & $-6,4375$ & 0 \\
\hline Moda & 16,5 & $-7,9375$ & 0 \\
\hline $\begin{array}{l}\text { Desviación } \\
\text { Estándar }\end{array}$ & 10,6216 & 1,3871 & 25,6498 \\
\hline Varianza & 112,8193 & 1,924 & 657,9142 \\
\hline Curtosis & 0,6608 & 10,034 & 34,4551 \\
\hline $\begin{array}{l}\text { Coef. } \\
\text { Asimetria }\end{array}$ & $-0,3848$ & 2,2668 & 0,7196 \\
\hline $\begin{array}{l}\text { Evento } \\
\text { TAB3 }\end{array}$ & $Z h$ & $Z D R$ & Kdp \\
\hline Media & 12,8053 & $-6,1858$ & 0,2314 \\
\hline Mediana & 13,5 & $-6,5625$ & 0 \\
\hline Moda & 15 & $-7,9375$ & 0 \\
\hline $\begin{array}{l}\text { Desviación } \\
\text { Estándar }\end{array}$ & 9,4779 & 1,5325 & 26,1457 \\
\hline Varianza & 89,8308 & 2,3485 & 683,5991 \\
\hline
\end{tabular}




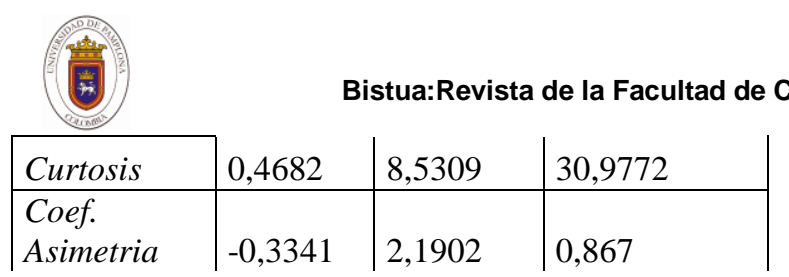

Los valores de todas las variables polarimétricas en el caso del radar de Corozal se encuentran entre los rangos estándar, no se presenta el mismo fenómeno que sucede con algunas variables del radar de Tablazo

Tabla 13.Caracterización estadística de los observables radar Corozal

\begin{tabular}{|c|c|c|c|}
\hline $\begin{array}{l}\text { Evento } \\
\text { COR2 }\end{array}$ & $Z h$ & $Z_{D R}$ & $K d p$ \\
\hline Media & 25,1259 & 2,291 & 0,1219 \\
\hline Mediana & 24,5 & 2 & 0 \\
\hline Moda & 23 & 1,3125 & 0 \\
\hline $\begin{array}{l}\text { Desviación } \\
\text { Estándar }\end{array}$ & 9,7648 & 2,0929 & 20,1055 \\
\hline Varianza & 95,3509 & 4,3801 & 404,231 \\
\hline Curtosis & 0,7056 & 7,7997 & 50,527 \\
\hline $\begin{array}{l}\text { Coef. } \\
\text { Asimetria }\end{array}$ & $-0,1035$ & $-1,5314$ & 1,383 \\
\hline $\begin{array}{l}\text { Evento } \\
\text { COR3 }\end{array}$ & $Z h$ & $Z_{D R}$ & $K d p$ \\
\hline Media & 25,663 & 3,0447 & 0,1105 \\
\hline Mediana & 26,5 & 3,0625 & 0 \\
\hline Moda & 28 & 2,25 & 0 \\
\hline $\begin{array}{l}\text { Desviación } \\
\text { Estándar }\end{array}$ & 7,5893 & 2,1522 & 21,9198 \\
\hline Varianza & 57,5981 & 4,6321 & 480,4774 \\
\hline Curtosis & 1,2616 & 8,9818 & 45,3377 \\
\hline $\begin{array}{l}\text { Coef. } \\
\text { Asimetria }\end{array}$ & $-0,5427$ & $-1,9304$ & 0,9983 \\
\hline
\end{tabular}

3.5. Análisis de los resultados de clasificadores de los fabricantes de los radares

Para el clasificador $\mathrm{C} 2$, los resultados totales son:

Tabla 14. Análisis Clasificador C2. Fuente: Elaboración propia

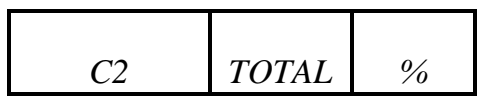

\begin{tabular}{|l|c|c|}
\hline 18.16(1):56-66 & \multicolumn{1}{l}{} \\
\hline Convectiva & 747260 & 83,83 \\
\hline Estratiforme & 134335 & 15,07 \\
\hline No usado & 9776 & 1,10 \\
\hline Prohibido & 0 & 0,00 \\
\hline Total & 891371 & 100 \\
\hline
\end{tabular}

Como se puede apreciar en la Tabla 14, la mayoría de celdas $(83.8 \%)$ tanto en Tablazo como en Corozal corresponden a eventos convectivos, el 15,07 \% a eventos estratiformes y un $1.1 \%$ no fue clasificado.

Para el caso del clasificador $\mathrm{C} 1$, los resultaos se muestran en la Tabla 15.

Tabla 15. Análisis Clasificador C1. Fuente: Elaboración Propia

\begin{tabular}{|l|c|c|}
\hline \multicolumn{1}{|c|}{ C1 } & TOTAL & $\%$ \\
\hline No disponible & 270 & 0,03 \\
\hline $\begin{array}{l}\text { Eco de } \\
\text { tierra/PA }\end{array}$ & 61484 & 6,90 \\
\hline $\begin{array}{l}\text { Dispersión } \\
\text { biologica }\end{array}$ & 72581 & 8,14 \\
\hline Precipitación & 0 & 0,00 \\
\hline $\begin{array}{l}\text { Gotas } \\
\text { Grandes }\end{array}$ & 0 & 0,00 \\
\hline $\begin{array}{l}\text { Precipitación } \\
\text { suave }\end{array}$ & 702884 & 78,85 \\
\hline $\begin{array}{l}\text { Precipitación } \\
\text { moderada }\end{array}$ & 46796 & 5,25 \\
\hline No usada & 7356 & 0,83 \\
\hline Total & 891371 & 100,00 \\
\hline
\end{tabular}

En este caso puntual se aprecia que en la mayoría de eventos predomina la precipitación suave con un $78.8 \%$ del total. En este caso también se observa una población significativa de celdas 
65

clasificadas como dispersiones biológicas y propagaciones anómalas para todos los eventos.

Para el caso del clasificador CO, los resultados se muestran en la Tabla 16.

Tabla 16. Análisis Clasificador Co. Fuente: Elaboración Propia

\begin{tabular}{|l|c|c|}
\hline \multicolumn{1}{|c|}{ CO } & TOTAL & $\%$ \\
\hline No disponible & 0 & 0,00 \\
\hline $\begin{array}{l}\text { Objetivo no } \\
\text { meteorológico }\end{array}$ & 134335 & 15,07 \\
\hline Lluvia & 648647 & 72,77 \\
\hline Nieve Húmeda & 50274 & 5,64 \\
\hline Nieve & 38529 & 4,32 \\
\hline Graupel & 14498 & 1,63 \\
\hline Granizo & 5088 & 0,57 \\
\hline No usada & 0 & 0,00 \\
\hline Total & 891371 & 100,00 \\
\hline
\end{tabular}

En este caso puntual se puede apreciar que una muestra significativa de celdas (11.6\%) en los diferentes eventos fueron clasificadas como nieve húmeda, nieve y Graupel hidrometeoros que por las latitudes Colombianas es imposible que se presente. Esto reitera la anomalía en configuración del radar Tablazo.

\section{Conclusiones}

Se mostró la manera de extraer, transformar, visualizar y caracterizar estadísticamente las variables polarimétricas de dos radares en el territorio colombiano.
El análisis estadístico permitió determinar que en el radar el Tablazo se están obteniendo mediciones erróneas en Zdr y KDP, lo cual puede deberse a una mala configuración del radar 0 en el almacenamiento de estos datos.

Esta caracterización de datos es una base fundamental de análisis para proponer y desarrollar métodos de corrección de errores inherentes en la medición de radares polarimétricos, teniendo en cuenta que si conoce el comportamiento estadístico de los datos y se confronta con la teoría, se pueden proponer métodos de corrección para los radares colombianos.

De manera similar, analizando los resultados de los clasificadores incluidos en el software del radar, se encontraron algunos errores en cuanto a esta clasificación, en especial que un $11 \%$ de los hidrometeoros clasificados corresponden a nieve y graupel que es imposible existan en la geografía colombiana. Es un desarrollo y trabajo futuro, proponer métodos de corrección de fallas debidos a distintos aspectos del radar.

\section{Agradecimientos}

A la Aeronáutica Civil que facilitó el acceso a los datos de radares.

\section{Referencias Bibliografícas}

\author{
Gomez Vargas, E., \& Obregon Neira, N. (2015). \\ MODELO PARA LA ESTIMACIÓN \\ CUANTITATIVA DE PRECIPITACIÓN \\ A PARTIR DE DATOS DE RADARES \\ POLARIMÉTRICOS. Pontificia
}


Universidad Javeriana, FACULTAD DE INGENIERIA - DOCTORADO EN INGENIERIA, Bogotá<.

Socorras Quintero, V., Gomez Vargas, E., \& Obregon Neira, N. (2014). Calibración de imágenes de radares meteorológicos. Tecnura, 18(41).

Rinehart, R. (2007). Radar for Meteorologist. Iowa: Rinehart.

Bringi, V., Thurai, M., \& Hannesen, R. (2007). Dual-Polarization Weather Radar Handbook. Neuss: Selex-Gematronik.

E. Gómez Vargas, Ingeniero Electrónico. Magister Universidad Distrital Francisco José de Caldas. Doctorado en Ingeniería Pontificia Universidad Javeriana. Profesor Asociado de la Universidad Distrital, Facultad de Ingenierías y Coordinador de la Maestría en Ciencias de la Información y las comunicaciones. https://orcid.org/00000003-4957-7313

G. Robayo, Ingeniero Electrónico Universidad Distrital FJC; Estudiante de Maestría en Ciencias de la Información y las Comunicaciones de la Universidad Distrital Francisco José de Caldas. https://orcid.org/0000-0002-2724-4318

\section{J. Camargo, Ingeniero Electrónico} Universidad Distrital FJC; Magister en Ciencias de la Información y las Comunicaciones de la Universidad Distrital Francisco José de Caldas. Docente de la Universidad Distrital Francisco José de Caldas. https://orcid.org/0000-0003-34831884
66

L. Cadena, Ingeniera Catastral .Universidad Distrital FJC. Magister en Ciencias de la Información y las Comunicaciones (2018) de la Universidad Distrital Francisco José de Caldas. Asistente de coordinación en la Maestría en Ciencias de la Información y las Comunicaciones. https://orcid.org/00000002-5077-128X

*Para citar este artículo: Gomez Vargas E.; Robayo G.;Camargo J.; Cadena L. . Meteorological Radar data analysis in Colombian Territory for diagnostics and error detection. Revista Bistua.2018.16(1):56-66

+ Autor para el envió de correspondencia y la solicitud de las separatas: Gomez Vargas E. Universidad Distrital FJC. egomezvargas@gmail.com

Recibido: Noviembre 25 de 2016

Aceptado:Enero 15 de 2018 